\title{
The Application of Computed Tomography Perfusion in the Alberta Stroke Program Early Computed Tomography Score for Endovascular Treatment of Acute Ischemic Stroke in the Anterior Circulation
}

\author{
Wei-Yong Zhang' \\ Shi-Feng Xiang' \\ Su-Jun Yang' \\ Yi-Ping $\mathrm{Wu}^{2}$ \\ Jun-Tao $\mathrm{Li}^{2}$ \\ Guo-Kun Liu' \\ Jian-Fei Li' \\ Wei-Wei Wang' \\ 'Department of CT/MRI, Handan Central \\ Hospital, Handan, 05600I, People's \\ Republic of China; ${ }^{2}$ Department of \\ Neurology, Handan Central Hospital, \\ Handan, 05600I, People's Republic of \\ China
}

\begin{abstract}
Objective: The present study investigated the predictive value of each perfusion parameter of the Alberta Stroke Program Early Computed Tomography Score (ASPECTS) in CT perfusion (CTP) imaging for the prognosis of endovascular treatment at the time of admission in patients with acute ischemic stroke in the anterior circulation.

Patients and Methods: The imaging data of 62 patients with acute ischemic stroke in the anterior circulation with an onset time of $6 \mathrm{~h}$ or less were retrospectively analyzed. All patients underwent the one-stop whole-brain dynamic volume four-dimensional (4D) CT angiography (CTA)-CTP and cranial magnetic resonance imaging (MRI) within seven days after treatment. The patients were divided into better and worse prognosis groups according to their clinical symptoms, combined with an MRI-ASPECTS score of $\leq 6$ within seven days after treatment. The observed perfusion parameters included cerebral blood flow (CBF)ASPECTS, cerebral blood volume (CBV)-ASPECTS, mean transit time (MTT)-ASPECTS, and time to peak (TTP)-ASPECTS. The difference in ASPECTS scores involving the CTP parameter, as well as diagnostic power, was compared between the two groups of patients.

Results: All CTP-ASPECTS scores negatively correlated with clinical prognosis. The higher the CTP-ASPECTS scores preceding treatment in patients with ischemic stroke in the anterior circulation, the better the prognosis. There were statistically significant differences in the scores of CBF-ASPECTS and CBV-ASPECTS between the two groups $(\mathrm{P}<$ $0.05)$. Receiver operating curve (ROC) analysis showed that the parameter with the largest area under the curve (AUC) was the CBF-ASPECTS score $(\mathrm{P}=0.003)$, with a sensitivity of $90.9 \%$, a specificity of $59.1 \%$, and an AUC of 0.806 , which was the most valuable prognostic predictor.
\end{abstract}

Conclusion: The CBF-ASPECTS score presented significant value as a primary indicator for predicting the outcome of endovascular treatment in patients with acute ischemic stroke in the anterior circulation, and it had good application prospects in clinical practice.

Keywords: X-ray computed tomography, perfusion imaging, Alberta Stroke Program Early Computed Tomography Score, stroke

\section{Introduction}

Domestic and foreign studies have verified that the Alberta Stroke Program Early Computed Tomography Score (ASPECTS) is a simple and rapid quantitative assessment for the extent of infarction and a predictor for clinical outcomes that can help in the screening of patients requiring endovascular treatment and
Department of CT/MRI, Handan Central Hospital, 15 Zhonghua South Street, Hanshan District, Handan, 05600I,

People's Republic of China

Tel +86 I3932096565

Email xiangshifeng99@tom.com 
determine their prognosis. ${ }^{1,2}$ However, the reliability of the non-contrast CT (NCCT)-ASPECTS remains controversial. In particular, in the diagnosis of ultra-early stroke, the NCCT-ASPECTS has relatively low diagnostic accuracy. Computed tomography perfusion (CTP) technology has been widely used in the treatment of ischemic stroke, but the application of CTP-ASPECTS to a CTP parameter chart remains in the research stage in China and abroad. ${ }^{1,3}$ One-stop, whole-brain dynamic volume CTP and fourdimensional (4D) CT angiography (CTA) techniques, combined with multi-parametric ASPECT scores including cerebral blood volume (CBV)-ASPECTS, cerebral blood flow (CBF)-ASPECTS, mean transit time (MTT)ASPECTS, and time-to-peak (TTP)-ASPECTS were employed in the present study to provide an image basis for determining the choice of endovascular therapy and prognosis for patients with acute ischemic stroke in the anterior circulation.

\section{Patients and Methods}

\section{General Data}

A total of 62 patients, admitted within $6 \mathrm{~h}$ to Handan Central Hospital (China) from January 2016 to December 2019 with cerebral infarction in the middle cerebral artery supply area due to severe stenosis or occlusion of the internal carotid artery or middle cerebral artery, and who received endovascular treatment, were retrospectively analyzed. There were 42 males and 20 females within an age range of 39-78 years old (average age, 56.6). Based on whether patients were conscious or not, changes in muscle strength, and magnetic resonance imaging (MRI) findings within one week, patients were divided into a better or worse prognosis group. The participant inclusion criteria were as follows: 1) all patients signed a written informed consent form to be included in the study; 2) patients with onset of the disease within 6 $\mathrm{h}$ and with no cerebral hemorrhage or brain tumor confirmed by non-contrast computerized tomography (NCCT); 3) patients whose CTA showed severe stenosis or occlusion of the internal carotid artery or middle cerebral artery; 4) patients who eventually received endovascular treatment; 5) patients with preoperative CTP suggestive of reduced cerebral blood flow (CBF). The exclusion criteria were as follows: 1) patients with severe cardiac and pulmonary diseases; 2) patients with an existing history of intracranial surgery, a large infarction, and allergic history to iodine contrast. This study was conducted with approval from the Ethics Committee of Handan Central Hospital. Written informed consent was obtained from all patients.

\section{Scanning Apparatus and Parameters}

Computed tomography perfusion (Toshiba Aquilion One 320CT, Tokyo, Japan) was conducted for all patients. During the examination, the patient was placed in the supine position with the head centered; the scanning range was $16 \mathrm{~cm}$ up from the base of the skull. A $20 \mathrm{G}$ cannula was inserted in the middle elbow vein using a dual-channel hyperbaric syringe; Uvexan (370 mg I/mL, Bayer, Germany) was used as a contrast agent. The 19-round volume scan protocol was divided into five sequences. The first sequence was delayed by $7 \mathrm{~s}$ to scan one round. The second sequence started at $10.7 \mathrm{~s}$ and scanned three rounds, with $2 \mathrm{~s}$ intervals per round. The third sequence started at $17 \mathrm{~s}$ and scanned six rounds with $2 \mathrm{~s}$ intervals per round. The fourth sequence started at $29.4 \mathrm{~s}$ and scanned four rounds with a $2 \mathrm{~s}$ interval per round. The fifth sequence started at $39.1 \mathrm{~s}$ and scanned five rounds with $5 \mathrm{~s}$ intervals per round. The tube voltage and current were $80 \mathrm{kV}$ and 300 $\mathrm{mA}$, respectively, and $60 \mathrm{~mL}$ of the contrast agent was injected at a flow velocity of $5 \mathrm{~mL} / \mathrm{s}$, followed by $40 \mathrm{~mL}$ of natural saline at the same velocity. Conventional scan sequences were adopted in the cranial MRI including T1WI and T2WI, MRI fluid-attenuated inversion recovery (FLAIR), and diffusion-weighted imaging (DWI). The scanning sequences were performed with the following parameters. T2WI adopts fast spin echo sequence: TR, $3770 \mathrm{~ms}$; TE, 95ms; FOV, 230mm; section thickness and spacing, 5mm and $1.5 \mathrm{~mm}$; scanning matrix, $320 \times 320 \mathrm{~mm}$; average value, 1; no parallel acquisition technology. T1WI adopts small angle excitation fast gradient echo sequence: TR, 180ms; TE, 2.71ms; flip angle, 70 ०; FOV, 230mm; section thickness and spacing, $5 \mathrm{~mm}$ and $1.5 \mathrm{~mm}$; scanning matrix, $320 \times 320 \mathrm{~mm}$; average value, 1 ; no parallel acquisition technology. Fluid attenuated T2WI sequence uses inversion recovery Fast spin Echo sequence TR, 8500ms; TE, 85ms; TI, 2440ms; FOV, 230ms; section thickness and spacing, $5 \mathrm{~mm}$ and $1.5 \mathrm{~mm}$; average value, 1 ; no parallel acquisition technology. The diffusion weighted imaging (DWI): TR and TE, $4500 \mathrm{~ms}$ and $74 \mathrm{~ms}$; b value, 1000; FOV, $230 \mathrm{~mm}$; section thickness and spacing, $5 \mathrm{~mm}$ and $1.5 \mathrm{~mm}$; matrix, $128 \times 128$; parallel acquisition factor, 2 ; average value, 2 . 


\section{Image Post-Processing}

All volume data were transferred to a Vitrea workstation for processing to obtain a CTP pseudo-color chart. The perfusion parameters included CBF, CBV, MTT, and TTP. Ten regions on two layers of the middle cerebral artery blood supply area were selected (Figure 1A-D) as follows: 1) the caudate nucleus and the layers below were divided into seven regions, ie, M1, M2, M3, insula (I), lenticular (L), caudate nucleus (C), and the posterior limb of the internal capsule (IC); 2) the cerebral cortex above the nucleus including the M4, M5, and M6 regions. The 10 regions have the same weight, all with 1 point. A total score of 10 points represented normal, and the total score was reduced by 1 point for each area affected. The four parameters CBF, CBV, MTT, and TTP were scored before the endovascular treatment, and MRI-DWI was scored within one week after the endovascular treatment. The 4D digital subtraction angiography (DSA) images were created after the automatic subtraction of cranial arteries using a workstation software package (Figure 1E).

\section{Statistical Analysis of the Perfusion \\ Parameters}

The SPSS Statistics 23.0 software was used to effect statistical analysis. Each parameter of the preoperative scores of the CTP-ASPECTS was compared between the good and worse prognosis groups using the rank-sum test. The receiver operating curve (ROC) was used to analyze the predictive efficacy of the four parameters, with the area under the curve (AUC) calculated together with the considered diagnostic accuracy, sensitivity, and specificity; $\mathrm{P}<0.05$ was considered to reflect a statistically significant difference.
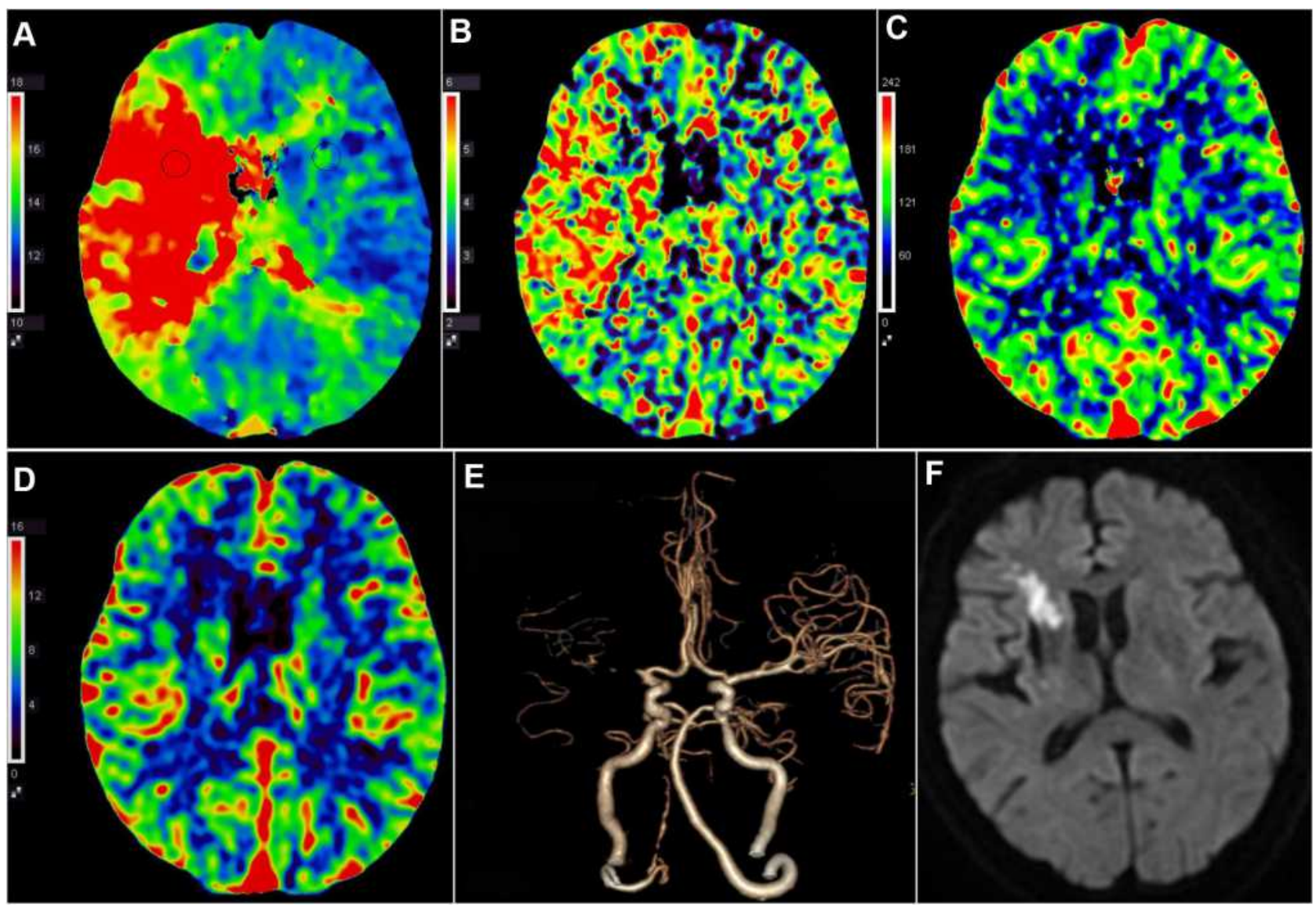

Figure I Case illustration of MRI-ASPECTS score. (A) TTP was prolonged in MI, M2, C, L and IC areas in right hemisphere than in corresponding contralateral brain tissue with TTP-ASPECTS score 5. (B) MTT was prolonged in MI, M2, C, L and IC areas in right hemisphere than in contralateral brain tissue with MTT-ASPECTS score 5. (C) The decrease of CBF in MI, C and IC areas in right hemisphere compared with contralateral corresponding areas with CBF-ASPECTS score 7. (D) The decrease of CBV in C and IC areas in right hemisphere compared with contralateral corresponding areas with CBV-ASPECTS score 8. (E) 4D-CTA showed the occlusion of right middle cerebral artery. (F) DWI demonstrated high signal intensity in C and IC areas in right hemisphere after 5 days of mechanical thrombectomy therapy with the MRI-ASPECTS score 8. The patients discharged with the recovery of the muscle strength of the left limb and can walk normally. 


\section{Results}

The Correlation Between the Baseline Pre-Operative Scores of CTP in the Alberta Stroke Program Early Computed Tomography Score and Prognosis

Based on whether the patient was conscious or not, the recovery of muscle strength, and MRI examination results within one week (Figure 1F), a total of 45 patients with no hemorrhage, no expansion of infarction, and an MRIASPECTS score $>6$ were included in the better prognosis group. A total of 17 patients with postoperative hemorrhage, expansion of infarction, and an MRI-ASPECTS score $\leq 6$ were included in the worse prognosis group. The mean values of the preoperative scores of CBF-ASPECTS, CBVASPECTS, MTT-ASPECTS, and TTP-ASPECTS were greater in the better prognosis group compared with the worse prognosis group, and a higher ASPECTS score was correlated with a good prognosis. When comparing the CTPASPECTS between the two groups, the results showed that the scores of CBF-ASPECTS $(Z=-2.949, P=0.003), \mathrm{CBV}-$ ASPECTS $(Z=-2.481, P=0.013)$, MTT-ASPECTS $(Z=-$ $1.242, \mathrm{P}=0.214)$, TTP-ASPECTS $(Z=-0.802, \mathrm{P}=0.422)$, and there existed a statistically significant difference in CBFASPECTS and CBV-ASPECTS between the better and the worse prognosis groups ( $\mathrm{P}<0.05)$. There was no statistically significant difference in the scores of MTT-ASPECTS and TTP-ASPECTS between the two groups $(\mathrm{P}>0.05)$ (Table 1).

\section{Analysis of the Prognostic ROC Predicted by the Preoperative Scores of CTP in the Alberta Stroke Program Early Computed Tomography Score}

The ROC analysis was conducted for CBF-ASPECTS, CBV-ASPECTS, MTT-ASPECTS, and TTP-ASPECTS scores. The results indicated that the AUC of the above parameters was $0.806,0.752,0.628$, and 0.583 , respectively. The AUC of the CBF-ASPECTS was the largest (Figure 2).
The preoperative CBF-ASPECTS had the best diagnostic efficacy in the semi-quantitative evaluation of the endovascular treatment in patients with acute ischemic stroke in the anterior circulation and was the most reliable indicator for postoperative clinical outcome. Using a score of 6 as the best cut-off point for prediction, CBF-ASPECTS $\leq 6$ indicated a sensitivity of $90.9 \%$ and specificity of $59.1 \%$.

\section{Discussion}

Approximately $28-46 \%$ of acute ischemic strokes in the anterior circulation are due to the occlusion of large vessels, often suggesting a poor prognosis. Accordingly, early treatment of the responsible vessels and the opening of obstructed vessels to save the ischemic penumbra are key to treatment. Currently, both domestic and foreign guidelines recommend endovascular therapy for ischemic stroke as having the highest level of evidence (Class I, Level A evidence). These recommendations were for the first time suggested for endovascular treatment represented by mechanical thrombectomy. ${ }^{4-6}$ Neuroimaging examination plays an important clinical role in assessing the severity of stroke and prognosis before surgery. ${ }^{5-9}$ The traditional scores of NCCT ASPECTS in patients with acute cerebral infarction show no remarkable image development in the early stage, and the diagnostic reliability of these scores is controversial and confusing for prognostic assessment. ${ }^{10,11}$ In the present study, the CTP-ASPECTS scoring method was applied to the endovascular treatment for acute ischemic stroke in the anterior circulation, and four ASPECT parameters were used to retrospectively analyze endovascular treatment in patients with acute ischemic stroke in the anterior circulation to better understand the reliable parameters for pre-operative neuroimaging diagnosis.

The four image parameters included the CBFASPECTS, CBV-ASPECTS, MTT-ASPECTS, and TTPASPECTS, produced within the CTP-ASPECTS. Each parameter had a different meaning in terms of evaluating the CBF. As such, it was necessary to screen the most

Table I The Results of the Scores of CTP-ASPECTS

\begin{tabular}{|l|l|l|l|l|l|}
\hline \multirow{2}{*}{ Parameters } & \multicolumn{2}{|l|}{ Good Prognosis (44) } & \multicolumn{2}{l|}{ Bad Prognosis (18) } & $\mathbf{Z}$ \\
\cline { 2 - 7 } & Median (M) & Inter-Quartile Range & Median (M) & Inter-Quartile Range & \\
\hline CBF & 7 & $6-7$ & 5 & $5-6$ & -2.949 \\
CBV & 7 & $6-8$ & 6 & $6-7$ & 0.003 \\
MTT & 5.5 & $5-6$ & 5 & $4-6$ & -2.481 \\
TTP & 5 & $5-6$ & 5 & $4-6$ & -1.013 \\
& & & & 0.214 \\
\end{tabular}




\section{ROC Curve}

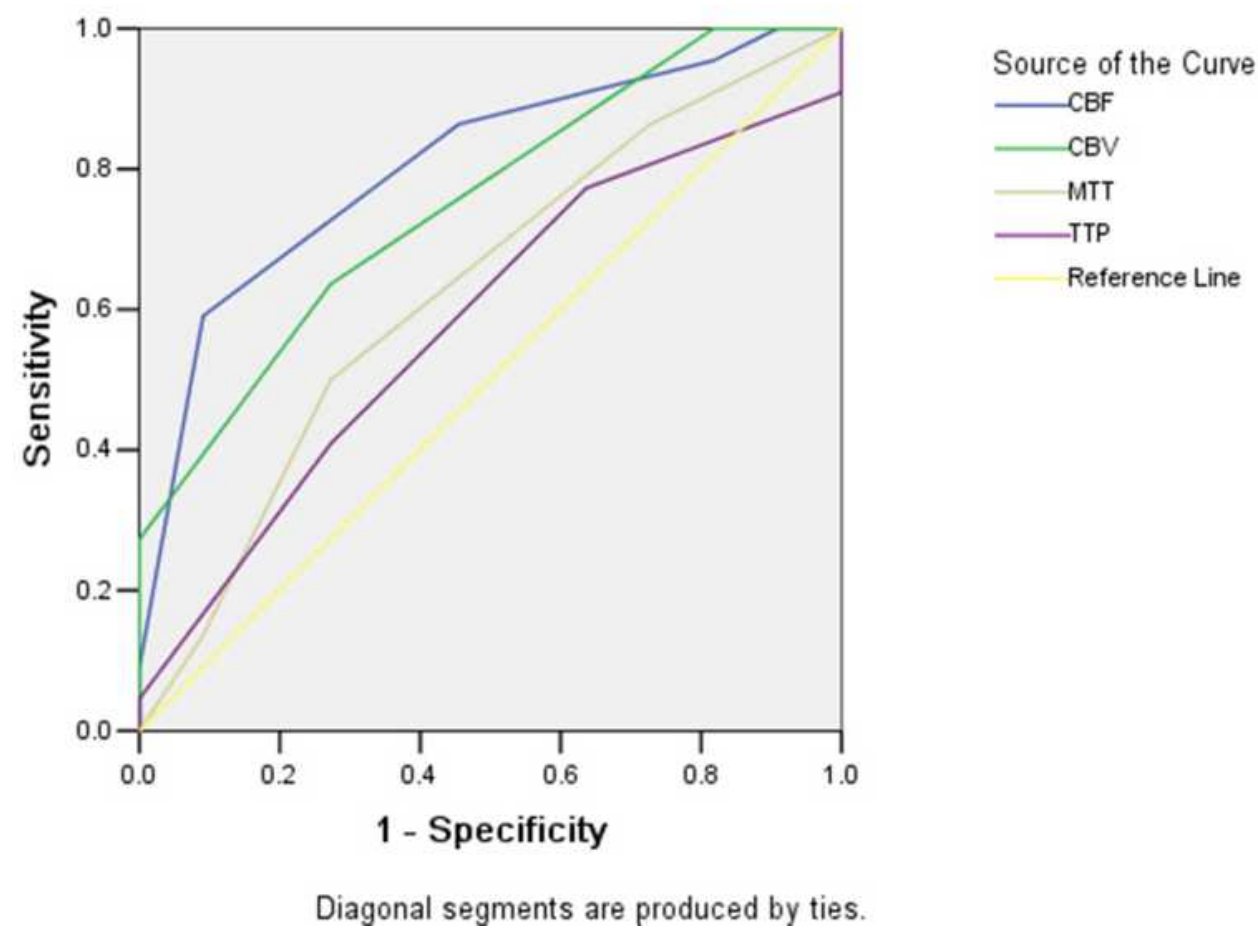

Figure 2 The receiver operating curves of the computed tomography Alberta Stroke Program Early Computed Tomography Score (ASPECTS) parameters including the cerebral blood flow, cerebral blood volume, mean transit time, and time to peak ASPECTS in the two groups of patients, with an area under curve of $0.806,0.752,0.628$, and 0.583 , respectively.

reliable image parameter to achieve the best prediction for postoperative prognosis. Rava et $\mathrm{al}^{12,13}$ have shown that CTP parameters can better distinguish the infarct core from the ischemic penumbra, and the mismatch of CBFASPECTS and CBV-ASPECTS can fully predict the volume of potentially salvage brain tissue. In the analysis conducted in the present study, no statistically significant difference was found in the MTT-ASPECTS and TTPASPECTS scores between the better and worse prognosis groups $(\mathrm{P}>0.05)$. These results indicated that MTT and TTP may reflect the average transit time and blood-flow velocity of blood passing through all the blood supply vessels in the brain tissue. Abnormalities in these parameters can be found even in the case of only a slight injury to cerebral blood flow. Furthermore, these parameters could not distinguish between ischemia, infarction core, and ischemic penumbra. There were statistically significant differences in the scores of CBF-ASPECTS and CBV-ASPECTS between the better and worse prognosis groups $(\mathrm{P}<0.05)$, and the parameters were higher in the better than the worse prognosis group. These results suggested that the region with decreased $\mathrm{CBF}$ and $\mathrm{CBV}$ may reflect the infarction core and ischemic penumbra, which could be adopted to determine the final approximate scope of infarction and salvageable ischemic brain tissue before surgery. The endovascular treatment of mechanical thrombectomy was less effective when the CBF-ASPECTS and CBV-ASPECTS had a score below 6 points, which was consistent with the results of domestic and foreign studies. ${ }^{14,15}$

The results of the present study show that the scores of CBF-ASPECTS and CBV-ASPECTS may reliably predict a poor prognosis in patients receiving endovascular treatment for acute ischemic stroke in the anterior circulation. Meanwhile, analysis of the ROC revealed that the AUC of the CBF-ASPECTS score was 0.806 , which was significantly higher compared with the score of CBV-ASPECTS (with an AUC of 0.752). Accordingly, the CBF-ASPECTS score was considered better suitable than the CBV-ASPECTS, MTTASPECTS, and TTP-ASPECTS scores for assessing the prognosis of endovascular treatment for acute stroke in the anterior circulation. The optimal cut-off point for the prediction has been inconsistently reported in China and abroad but is still in the research stage. ${ }^{16,17}$ In the present study, a CBF- 
ASPECTS of $\leq 6$ was adopted as the optimal cut-off point for suggesting a poor prognosis in terms of clinical outcome. This was because CBF may have been the most representative parameter for cerebral ischemia. A reduced $\mathrm{CBF}$ may lead to cerebral oxygen metabolism disorder; if the obstructed blood vessels can be revascularized in time, however, blood flow will be restored in the case of cerebral ischemia and cerebral infarction, and good therapeutic results after surgery can be achieved. Moreover, the 320-row whole-brain CTP can obtain 4D-CTA images to help further understand the site of the responsible vascular lesion, and facilitate the further development of endovascular treatment protocols. ${ }^{18-20}$

Our study had some limitations, such as the small sample size and the relatively short follow-up time. Further expansion of sample size, longer follow-up duration, and multicenter studies are needed.

In summary, CTP-ASPECTS scores may be useful for the early prognostic assessment of endovascular treatment for acute ischemic stroke in the anterior circulation. Furthermore, CBF-ASPECTS score may be more reliable and deserve further clinical application.

\section{Ethics Approval and Consent to Participate}

This study was conducted with approval from the Ethics Committee of Handan Central Hospital. This study was conducted in accordance with the declaration of Helsinki. Written informed consent was obtained from all patients.

\section{Funding}

No external funding received to conduct this study.

\section{Disclosure}

The authors declare that they have no competing interests.

\section{References}

1. Lassalle L, Turc G, Tisserand M, et al. ASPECTS (Alberta Stroke Program Early CT Score) assessment of the perfusion-diffusion mismatch. Stroke. 2016;47(10):2553-2558. doi:10.1161/ STROKEAHA.116.013676

2. Haussen DC, Dehkharghani S, Rangaraju S, et al. Automated CT perfusion isehemie core volume and noneontrast CT ASPECTS (Alberta Stroke Program Early CT Score): correlation and clinical outcome prediction in large vessel stroke. Stroke. 2016;47 (9):2318-2322. doi:10.1161/STROKEAHA.116.014117

3. Schroder J, Thomalla G. A critical review of alberta stroke program early ct score for evaluation of acute stroke imaging. Front Neurol. 2017;12(7):245.

4. Campbell BC, Mitchell PJ, Kleinig TJ, et al. Endovascular therapy for ischemic stroke with perfusion-imaging selection. $N$ Engl $\mathrm{J} \mathrm{Med}$. 2015;372(11):1009-1018. doi:10.1056/NEJMoa1414792
5. Goyal M, Demchuk AM, Menon BK, et al. Randomized assessment of rapid endovascular treatment of ischemic stroke. $N$ Engl J Med. 2015;372(11):1019-1030. doi:10.1056/NEJMoa1414905

6. Jovin TG, Chamorro A, Cobo E, et al. Thrombectomy within 8 hours after symptom onset in ischemic stroke. $N$ Engl J Med. 2015;372 (24):2296-2306. doi:10.1056/NEJMoa1503780

7. Saver JL, Goyal M, Bonafe A, et al. Stent-retriever thrombectomy after intravenous t-pa vs. T-pa alone in stroke. $N$ Engl $J$ Med. 2015;372(24):2285-2295. doi:10.1056/NEJMoa1415061

8. Parsons MW, Pepper EM, Bateman GA, Wang Y, Levi CR. Identification of the penumbra and infarct core on hyperacute noncontrast and perfusion ct. Neurology. 2007;68(10):730-736. doi:10.1212/01.wnl.0000256366.86353.ff

9. Lin K, Rapalino O, Lee B, et al. Correlation of volumetric mismatch and mismatch of alberta stroke program early ct scores on ct perfusion maps. Neuroradiology. 2009;51(1):17-23. doi:10.1007/s00234008-0454-y

10. Padroni M, Bernardoni A, Tamborino C, et al. Cerebral blood volume aspects is the best predictor of clinical outcome in acute ischemic stroke: a retrospective, combined semi-quantitative and quantitative assessment. PLoS One. 2016;11(1):e147910. doi:10.1371/journal. pone. 0147910

11. Sillanpaa N, Saarinen JT, Rusanen H, et al. Ct perfusion aspects in the evaluation of acute ischemic stroke: thrombolytic therapy perspective. Cerebrovasc Dis Extra. 2011;1(1):6-16. doi:10.1159/ 000324324

12. Rava RA, Snyder KV, Mokin M, et al. Assessment of a Bayesian Vitrea CT perfusion analysis to predict final infarct and penumbra volumes in patients with acute ischemic stroke: a comparison with RAPID. AJNR Am J Neuroradiol. 2020;41(2):206-212. doi:10.3174/ ajnr.A6395

13. Rava RA, Snyder KV, Mokin M, et al. Assessment of computed tomography perfusion software in predicting spatial location and volume of infarct in acute ischemic stroke patients: a comparison of Sphere, Vitrea, and RAPID. J Neurointerv Surg. 2021;13(2):130-135. doi:10.1136/neurintsurg-2020-015966

14. van Seeters T, Biessels GJ, Kappelle LJ, et al. The prognostic value of ct angiography and ct perfusion in acute ischemic stroke. Cerebrovasc Dis. 2015;40(5-6):258-269. doi:10.1159/000441088

15. Penumbra Pivotal Stroke Trial I. The penumbra pivotal stroke trial: safety and effectiveness of a new generation of mechanical devices for clot removal in intracranial large vessel occlusive disease. Stroke. 2009;40(8):2761-2768. doi:10.1161/STROKEAHA.108.544957

16. Yoo AJ, Zaidat OO, Chaudhry ZA, et al. Impact of pretreatment noncontrast ct alberta stroke program early ct score on clinical outcome after intra-arterial stroke therapy. Stroke. 2014;45(3):746-751. doi:10.1161/STROKEAHA.113.004260

17. Liebeskind DS, Jahan R, Nogueira RG, et al. Serial Alberta Stroke Program Early CT Score from baseline to 24 hours in solitaire flow restoration with the intention for thrombectomy study. Stroke. 2014;45(3):723-727. doi:10.1161/STROKEAHA.113.003914

18. Jiang L, Chen HY, Yin XD, et al. [Prognostic value of dwi-aspects in patients with acute cerebral infarction after intravenous thrombolytic therapy]. Lin Chuang Fang She Xue Za Zhi. 2016;35(3):328-332. (Chinese).

19. Raza SA, Barreira CM, Rodrigues GM, et al. Prognostic importance of CT ASPECTS and CT perfusion measures of infarction in anterior emergent large vessel occlusions. J Neurointerv Surg. 2019;11 (7):670-674. doi:10.1136/neurintsurg-2018-014461

20. Dong LJ, Wang T, Yang C, et al. [Clinical application of dynamic cerebral artery CTA based on whole brain CT perfusion data in cerebrovascular diseases]. Lin Chuang Fang She Xue Za Zhi. 2018;37(1):148-153. (Chinese). 


\section{Publish your work in this journal}

The International Journal of General Medicine is an international, peer-reviewed open-access journal that focuses on general and internal medicine, pathogenesis, epidemiology, diagnosis, monitoring and treatment protocols. The journal is characterized by the rapid reporting of reviews, original research and clinical studies

across all disease areas. The manuscript management system is completely online and includes a very quick and fair peer-review system, which is all easy to use. Visit http://www.dovepress.com/ testimonials.php to read real quotes from published authors. 